\title{
Metastatic breast cancer patient perceptions of somatic tumor genomic testing
}

Elizabeth J. Adams ${ }^{1+}$, Sarah Asad ${ }^{1 \dagger}$, Raquel Reinbolt ${ }^{2,3}$, Katharine A. Collier ${ }^{1,2}$, Mahmoud Abdel-Rasoull, Susan Gillespie ${ }^{2,5}$, James L. Chen ${ }^{1,2}$, Mathew A. Cherian ${ }^{1,2,5}$, Anne M. Noonan ${ }^{1,2}$, Sagar Sardesai 1,2,5, Jeffrey VanDeusen ${ }^{1,2,5}$, Robert Wesolowski ${ }^{1,2,5}$, Nicole Williams ${ }^{1,2,5}$, Charles L. Shapiro ${ }^{6}$, Erin R. Macrae ${ }^{7}$, Robert Pilarski ${ }^{5,8}$, Amanda E. Toland ${ }^{8}$, Leigha Senter ${ }^{5,8}$, Bhuvaneswari Ramaswamy ${ }^{1,2,5}$, Clara N. Lee ${ }^{1,5,9,10 \dagger}$, Maryam B. Lustberg ${ }^{1,2,5+}$ and Daniel G. Stover ${ }^{1,2,5,11^{* \dagger}}$ (i)

\begin{abstract}
Background: To assess metastatic breast cancer (MBC) patient psychological factors, perceptions, and comprehension of tumor genomic testing.

Methods: In a prospective, single institution, single-arm trial, patients with MBC underwent next-generation sequencing at study entry with sequencing results released at progression. Patients who completed surveys before undergoing sequencing were included in the present secondary analysis $(n=58)$. We administered four validated psychosocial measures: Center for Epidemiologic Studies Depression Scale, Beck Anxiety Inventory, Trust in Physician Scale, and Communication and Attitudinal Self-Efficacy scale for Cancer. Genetic comprehension was assessed using 7-question objective and 6-question subjective measures. Longitudinal data were assessed $(n=40)$ using paired Wilcoxon signed rank and McNemar's test of agreement.

Results: There were no significant differences between the beginning and end of study in depression, anxiety, physician trust, or self-efficacy (median time on study: 7.6 months). Depression and anxiety were positively associated with each other and both negatively associated with self-efficacy. Self-efficacy decreased from pre- to post-genomic testing $(p=0.05)$. Objective genetics comprehension did not significantly change from pre- to postgenomic testing, but patients expressed increased confidence in their ability to teach others about genetics $(p=$ 0.04). Objective comprehension was significantly lower in non-white patients $(p=0.02)$ and patients with lower income $(p=0.04)$.

(Continued on next page)
\end{abstract}

\footnotetext{
* Correspondence: daniel.stover@osumc.edu

Prior Presentations: This work was presented at the San Antonio Breast Cancer Symposium 2019

Clara N. Lee, Maryam B. Lustberg and Daniel G. Stover indicates authors equally directed the work.

'The Ohio State University Comprehensive Cancer Center, Arthur G. James Cancer Hospital and Richard J. Solove Research Institute, Columbus, OH, USA

${ }^{2}$ Division of Medical Oncology, Ohio State University College of Medicine, Columbus, OH, USA

Full list of author information is available at the end of the article
}

(c) The Author(s). 2020 Open Access This article is licensed under a Creative Commons Attribution 4.0 International License, which permits use, sharing, adaptation, distribution and reproduction in any medium or format, as long as you give appropriate credit to the original author(s) and the source, provide a link to the Creative Commons licence, and indicate if changes were made. The images or other third party material in this article are included in the article's Creative Commons licence, unless indicated otherwise in a credit line to the material. If material is not included in the article's Creative Commons licence and your intended use is not permitted by statutory regulation or exceeds the permitted use, you will need to obtain permission directly from the copyright holder. To view a copy of this licence, visit http://creativecommons.org/licenses/by/4.0/. The Creative Commons Public Domain Dedication waiver (http://creativecommons.org/publicdomain/zero/1.0/) applies to the data made available in this article, unless otherwise stated in a credit line to the data. 
(Continued from previous page)

Conclusions: This is the only study, to our knowledge, to longitudinally evaluate multiple psychological metrics in $\mathrm{MBC}$ as patients undergo tumor genomic testing. Overall, psychological dimensions remained stable over the duration of tumor genomic testing. Among patients with $\mathrm{MBC}$, depression and anxiety metrics were negatively correlated with patient self-efficacy. Patients undergoing somatic genomic testing had limited genomic knowledge, which varied by demographic groups and may warrant additional educational intervention.

Clinical trial information: NCT01987726, registered November 13, 2013.

Keywords: Metastatic breast cancer, Genomics

\section{Background}

More than 150,000 women are living with metastatic breast cancer $(\mathrm{MBC})$ in the United States alone [1]. Because of increasing evidence that specific somatic tumor mutations are both prognostic (e.g. ESR1 [2-4]) and predictive in MBC (e.g. ESR1 [3] and PIK3CA [4-6]), somatic genomic testing has become standard of care and offers great promise in the advancement of novel therapeutics and precision cancer medicine [7-10]. Despite the richness of information next-generation sequencing (NGS) offers clinicians, the effects of genomic testing, particularly somatic tumor sequencing, on patient psychosocial outcomes and perceptions of care is understudied [11-14].

While the promise of genomic testing remains significant, absence of detected targetable mutations or inaccessibility to clinical trials based on detected targetable mutations may increase negative emotions in the metastatic cancer population $[14,15]$. Patients note struggles with "information overload" and psychological distress due to cancer interfering with their ability to comprehend somatic tumor genomic testing $[16,17]$. Together, these studies support further investigation of patient psychological factors such as depression and anxiety, as well as trust in physician and self-efficacy (confidence in one's own ability to behave in a way that renders the results one desires, i.e., being able to seek and subsequently find information) in those undergoing somatic genomic testing. With recent widespread implementation of tumor genomic testing in $\mathrm{MBC}$, it is imperative to understand the psychosocial impact of somatic genomic testing on patients, patient comprehension of the genomic testing, patient perceptions of tumor genomic testing, as well as the patient demographic characteristics that may mediate these factors [13].

We previously completed a prospective, single institution, single-arm trial, in which patients with $\mathrm{MBC}$ underwent NGS using Foundation Medicine (FM) to evaluate somatic cell mutations associated with 315 cancer-related genes [18] at study entry, with sequencing results released to providers at time of progression. We previously found that NGS impacted clinical decisionmaking in a minority of patients, and patients whose next cancer treatment was not supported by the genomic test had poorer perceptions of their care [19]. While several studies have reported the nuanced psychosocial effects of learning of one's harbored mutations from genetic testing using a longitudinal study design, all that we have been able to find have focused on germline sequencing [20-22], with a paucity of data regarding somatic genomic testing. The longitudinal study design in these studies has allowed for comparison from before to after the genomic testing, a critical component required to hone in on the influence of somatic testing on dimensions of patients' lives including psychological characteristics [15, 20-22].

We sought to evaluate patient psychosocial characteristics, genetic comprehension, and perceived risks and expectations of somatic cell NGS genomic testing longitudinally in the MBC setting in this secondary analysis. The primary objectives of this study were to 1) longitudinally describe patient psychological health before and after undergoing tumor NGS; 2) evaluate the relationship between psychological health and whether the patient underwent a FM-supported treatment; 3) assess patient comprehension of genetics and patient perceptions of tumor somatic genomic testing; 4) describe the relationship between patient psychosocial outcomes and sociocultural background.

\section{Methods \\ Study population}

This was a secondary analysis of data obtained from a prospective, single-site, single-arm trial at an NCIdesignated comprehensive cancer center. Patients with $\mathrm{MBC}$ who were within 10 weeks of starting their current line of therapy and had an estimated survival of $\geq 3$ months were included in this study. Participants also needed to have a tumor sample (primary or metastatic) available for genomic testing. The study population along with a description of the genomic testing has been previously described [19]. In the present manuscript, the study population includes participants who completed the pre-test (prior to genomic testing) questionnaire at study entry $(n=58)$ and/or the post-test questionnaire at the end of study visit $(n=40)$. All patients completed 
informed consent approved by the Ohio State University Institutional Review Board, which included a description of the FM somatic genomic testing and a discussion of FM's risks and benefits with their treating physician. While a formal script for physicians to use was not created for this discussion of the FM testing, physicians were required to cover pre-specified points including the capabilities and limitations of FM testing in terms of their care. This does introduce variance in how FM was discussed, however this realistically mirrors the MBC clinical setting, a goal of this study. Genomic test results were reviewed with participants; there was not a specific script for interactions or education provided beyond standard provider discussion.

\section{Survey measures}

Patient surveys included questions about mood, attitudes, and knowledge relating to cancer care and genomic testing. Four validated measures were used, including: Center for Epidemiologic Studies Depression Scale (CES-D) [23], Beck Anxiety Inventory (BAI) [24], Trust in Physicians/Providers Scale (TPS) [25], and Communication and Attitudinal Self-Efficacy scale for cancer (CASE-cancer) [26]. The CES-D utilizes 20 items to evaluate clinical depression [23] to determine the prevalence of depressive ideologies in the participant's preceding week. Response options to each item are 0 (rarely or none of the time), 1 (some or little of the time), 2 (moderately or much of the time), or 3 (most or all of the time). CES-D scores range from 0 to 60 with a score of 16 or higher indicating clinical depression [23]. The 21-question Beck Anxiety Inventory (BAI) [24] assesses the extent of bothersome anxiety symptoms in the past month using a 4-point Likert scale. Response options to each item are 0 (not at all bothered), 1 (mildly bothered), 2 (moderately bothered), and 3 (severely bothered). BAI scores range from 0 to 63 with a score from 0 to 9 indicating no anxiety, 10-18 indicating mild to moderate anxiety, and a score of 19 or higher indicating moderate to severe anxiety [24].

The 11-item Trust in Physician Survey (TPS) [25] uses a 5-point Likert scale that includes responses of agreement ranging from 0 (strongly disagree) to 4 (strongly agree). TPS scores range from 11 to 55 with higher scores indicating greater trust in physician [25]. The Communication and Self-Efficacy scale for cancer (CASE-cancer) evaluates patients' confidence in maneuvering through their cancer care and relationship with their medical oncologist [26]. CASE-cancer utilizes a 5point Likert scale ranging from 1 (strongly disagree) to 5 (strongly agree) to assess agreement with 12 items relating to patient self-efficacy. CASE-cancer scores range from 12 to 48 with higher scores indicating greater selfefficacy [26]. Patients who skipped more than two questions on a given measure did not have a score calculated.

Subjective and objective genetic knowledge were measured with items developed for this study. The response options for the 6 subjective questions were a 5-point Likert scale from strongly disagree to strongly agree. Objective knowledge was measured with 15 true or false statements designed to test patients' objective understanding of genes and genetics. We selected the seven statements about basic genetic information to assess. Examples of these statements include "It is possible to see a gene with the naked eye", "A gene is a piece of DNA", and "A person's race and ethnicity can affect how likely they are to get a disease". Questions included in the objective knowledge measure were based on a previously published genetic knowledge survey [27].

The only survey questions that were discussed with patients upon completing the pre-test and post-test surveys were when a patient reported moderate to severe psychological distress. In these cases, the patient was immediately referred to a social worker and to their primary care team. Genetic comprehension questions were not discussed with participants upon completion.

\section{Statistical analyses}

One-way ANOVA tests were used to assess pre-test CESD, BAI, TPS, and CASE-Cancer scores by self-reported income, education, insurance, and breast cancer receptor subtype. An independent $\mathrm{t}$-test was used to evaluate each patient reported outcome by race (white versus nonwhite). Paired Wilcoxon signed-rank tests were used to compare pre- and post-test scores. McNemar's test of agreement was used to compare pre- and post-test subjective knowledge about genetics. Due to our limited sample size, these questions were assessed as a 3-level outcome (agree vs. neutral vs. disagree). The objective knowledge questions were scored like a test ("gene test" score $=$ number of statements answered correctly/7) and combined into a continuous variable. Linear mixed effects models were fit to assess the association between treatment change and post-test scores for each of the four outcomes. These models included main effects of treatment change (no change versus change), time (pre vs. post), the interaction of treatment change with time, and random intercepts to account for repeated measures. Finally, Pearson's $\mathrm{r}$ correlation tests were performed to assess the associations among the outcomes. All figures were created in $\mathrm{R}$ version 3.4.1. All statistical analyses were performed using SAS version 9.4 and $\mathrm{R}$ version 3.4.1.

\section{Results}

Study population

As described previously [19], a total of 100 patients had successful FM NGS testing and were eligible for analysis 
(Fig. 1). Of these, 58 patients completed at least a portion of the pre-test, and 40 completed at least a portion of both the pre-test and post-test surveys. Eleven patients who completed the pre-test survey did not have their FM reports released and thus did not complete a post-test survey because they did not progress on their first treatment. We assessed for potential response bias and found that patients who completed the pre-test only did not differ from those who completed both surveys in terms of demographics such as age, race, breast cancer type, or ECOG status. The 58 patients who completed the pre-test survey were mostly aged 45-64 (65\%), white (81\%), ER+ (61\%), in the highest income (59\%) group, and had private insurance (73\%) (Table 1).

\section{Depression, anxiety, physician trust, and self-efficacy measures}

In the pre-test, patients had a mean CES-D score of 13.6 (range: 0-34) and a mean BAI score of 11.3 (range: 040 ). Categorically, $38 \%$ of patients were depressed (CES$\mathrm{D} \geq 16$ ), $28 \%$ of patients had mild to moderate anxiety (BAI 10-18), and 19\% of patients had moderate to severe anxiety $(B A I \geq 19)$. Patients had a mean TPS score of 48.4 (range: 34-55), indicating substantial trust in their medical oncologist. Patients had a mean CASE- cancer score of 42.4 (range: 16-48), indicating relatively high self-efficacy in navigation of their cancer care, specifically within the domains of finding and obtaining information, understanding and participating in their cancer care, and maintaining a positive attitude. There were no significant differences in any of the outcomes by income (all ANOVA $p>0.05$ ), education (all ANOVA $p>0.05$ ), race (all t-test $p>0.05$ ), insurance (all ANOVA $p>0.05$ ), or breast cancer type (all ANOVA $p>0.05$ ) (Supplementary Figure 1).

From pre-test (study entry) to post-test (end of study), there was no significant change in CES-D score (Wilcoxon $p$-value $=0.13$ ), BAI score (Wilcoxon $p$ value $=0.50)$, TPS score (Wilcoxon $\mathrm{p}$-value $=0.15$ ), while CASE-cancer score decreased from pre- to post-test, marginally non-significant (Wilcoxon p-value $=0.05$ ) (Fig. 2a). Finally, treatment change was not a significant predictor of post-test CES-D score, BAI score, TPS score, or CASE-cancer score in linear mixed model analyses (Supplementary Table 1).

We assessed correlations between our primary outcomes including CES-D, BAI, CASE-cancer, and TPS scores (Fig. 2b/c). Both in the pre-test and in the posttest, patients' CES-D scores were positively correlated with their BAI scores (Pearson's $r=0.61$ and 0.60 for

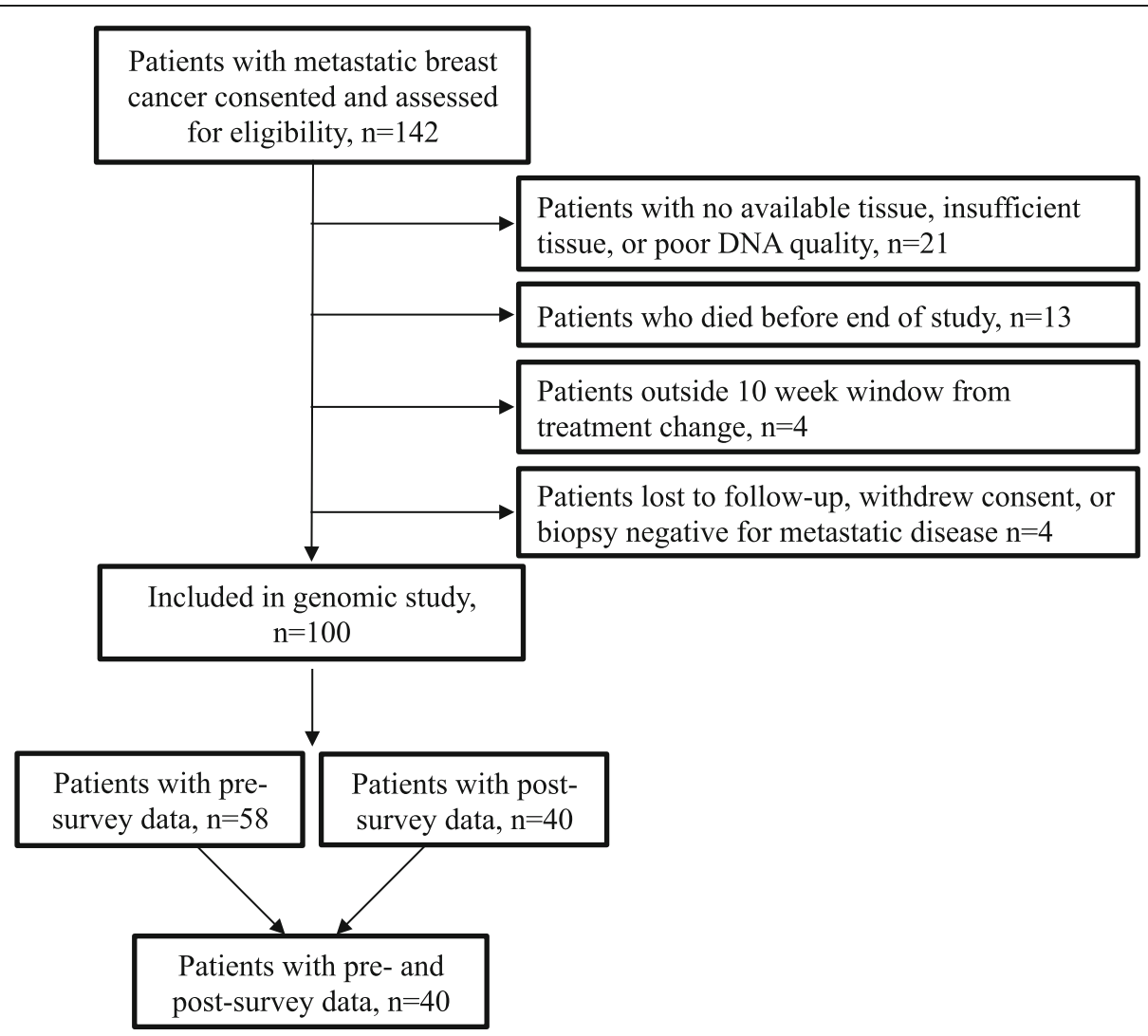

Fig. 1 CONSORT Diagram 
Table 1 Cohort Characteristics $(N=58)$

\begin{tabular}{|c|c|c|}
\hline & $\mathrm{N}$ & $\%$ \\
\hline \multicolumn{3}{|l|}{ Age } \\
\hline$<45$ & 7 & 12.1 \\
\hline $45-54$ & 19 & 32.8 \\
\hline $55-64$ & 17 & 29.3 \\
\hline$\geq 65$ & 15 & 25.9 \\
\hline \multicolumn{3}{|l|}{ Subtype } \\
\hline ER and/or PR+, HER2- & 35 & 61.4 \\
\hline HER2+ & 3 & 5.3 \\
\hline TNBC & 19 & 33.3 \\
\hline \multicolumn{3}{|l|}{ Race } \\
\hline Non-white ${ }^{a}$ & 11 & 19.0 \\
\hline White & 47 & 81.0 \\
\hline \multicolumn{3}{|l|}{ Education } \\
\hline High school diploma/GED or lower & 17 & 29.3 \\
\hline Some college, technical school, or Associate's degree & 20 & 34.5 \\
\hline Bachelor's degree or higher & 21 & 36.2 \\
\hline \multicolumn{3}{|l|}{ Income } \\
\hline$<\$ 40,000$ & 10 & 21.7 \\
\hline$\$ 40,000-\$ 69,999$ & 9 & 19.6 \\
\hline$\$ 70,000$ or more & 27 & 58.7 \\
\hline \multicolumn{3}{|l|}{ Insurance type } \\
\hline Medicaid or Medicare & 12 & 21.4 \\
\hline Private & 41 & 73.2 \\
\hline Other & 3 & 5.4 \\
\hline \multicolumn{3}{|l|}{ Treatment change based on FM test } \\
\hline No & 41 & 87.2 \\
\hline Yes & 6 & 12.8 \\
\hline
\end{tabular}

Number of prior therapies in metastatic setting

Median (range)

$1 \quad(0,11)$

${ }^{a}$ Non-white participants included those who self-identified as Black $(n=7$,

$14.6 \%)$, Asian ( $n=2,4.2 \%)$, Pacific Islander $(n=1,2.1 \%)$, and Multiracial

$(n=1,2.1 \%)$

pre-test and post-test, respectively; both $p<0.0001$ ). Patients' CASE-cancer scores were negatively correlated with CES-D and BAI in both the pre-test (Pearson's $\mathrm{r}=$ -0.43 and -0.42 for CES-D and BAI pre-test, respectively; both $p=0.001$ ) and the post-test (Pearson's $\mathrm{r}=-0.54$ and -0.37 for CES-D and BAI post-test; $p=$ 0.0004 and 0.03 , respectively).

\section{Subjective and objective genetic knowledge}

Six subjective genetic knowledge questions were compared between pre-test and post-test (Table 2). For questions 1-5, most patients agreed with the statements indicating high confidence in understanding genetic information. For these five questions, there were no significant changes from pre- to post-test. However, for question 6 about ability to explain to others how genes affect health, patients were equally spread across response values in the pre-test. For this question, there was a significant change from pre- to post-test ( $\mathrm{McNe}$ mars $p$-value $=0.04)$. In the post-test, significantly more patients (18 vs. 13 in the pre-test) agreed that they would be able to explain to others how genes affect health.

For objective knowledge, patients had a mean "genetic knowledge test" score of 0.72 (range: $0-1$ ) in the pre-test (Table 3). "Genetic knowledge test" score was significantly different by income group (ANOVA $p$-value $=$ $0.001)$ and race (t-test $p$-value $=0.04)$ yet did not differ significantly by age (ANOVA $p$-value $=0.55$ ), education (ANOVA $p$-value $=0.15$ ), or insurance (ANOVA $p$ value $=0.06$ ). Patients in the highest income group had significantly higher mean scores, and white women had significantly higher mean test scores than non-white women, while patients with private insurance had a nonsignificant numerically higher score. There was no significant change in score from pre- to post-test (Wilcoxon $p$-value $=0.57$; Fig. 3). "Genetic knowledge" was not significantly correlated with any of the four validated psychological measures (data not shown). We assessed correlations between CES-D, BAI, CASEcancer, TPS, "genetic knowledge," and number of FM recommended therapies in the post-test survey. Patients had a mean of 13.3 (range: 1-36) recommended therapies. We found no significant correlations between number of therapies with CES-D, BAI, CASE-cancer, TPS, or "genetic knowledge".

\section{Patient motivation, perception, and information seeking behavior}

We administered 9 questions to evaluate patient motivations and perceived risks and benefits of participation in the study (Supplementary Table 2). 32.7\% (18/55) of patients believed that finding out their cancer had a high chance of progressing would be too much to handle emotionally. However, most patients $(85.7 \%$; 48/56) did not agree that information about their cancer was best left unknown. Most patients were not concerned (58.9\%; 33/56) about FM testing being new. Furthermore, most patients $(77.4 \%$; $41 / 53)$ did not believe they would lose their job if the genomic testing results got out. About half of patients $(56.4 \%$; $31 / 55)$ believed the FM results would help them change behaviors to reduce disease risk. Most patients also believed $(63.6 \% ; 35 / 55)$ that the results would help them to seek medical attention to reduce disease risk. To assess information seeking behavior, we administered one question asking patients what steps they have taken to learn more after learning their study results. Most patients (85\%) sought information after their genomic testing. 57.5\% of patients asked their 

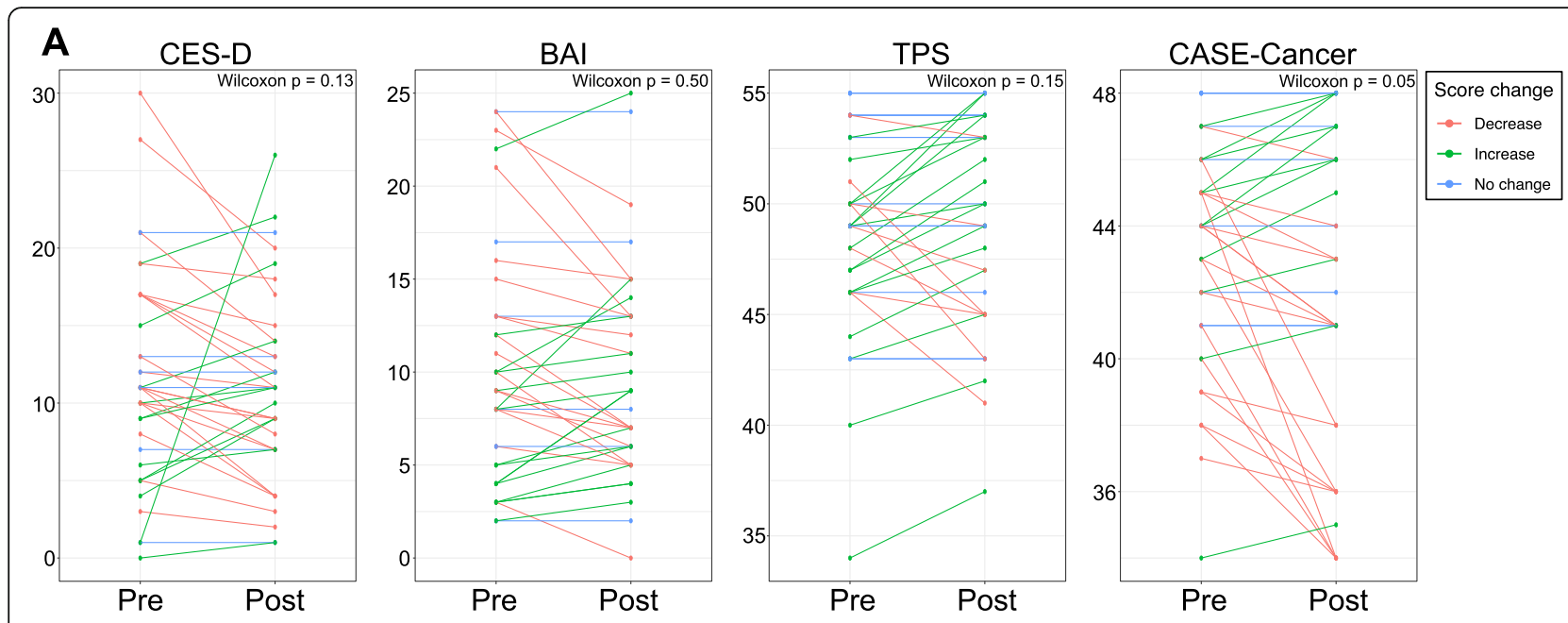

B

\section{Pre-Survey}

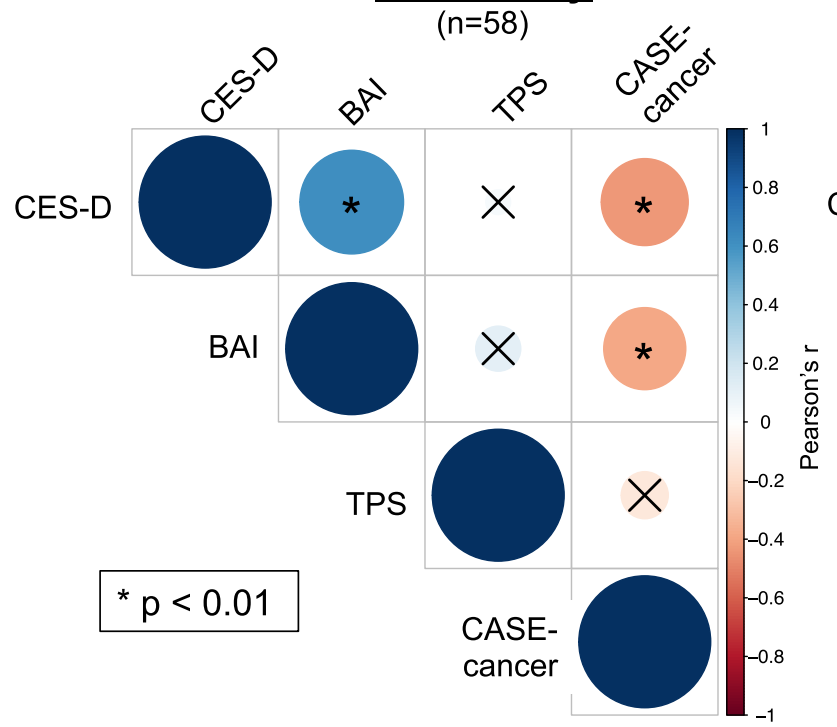

C

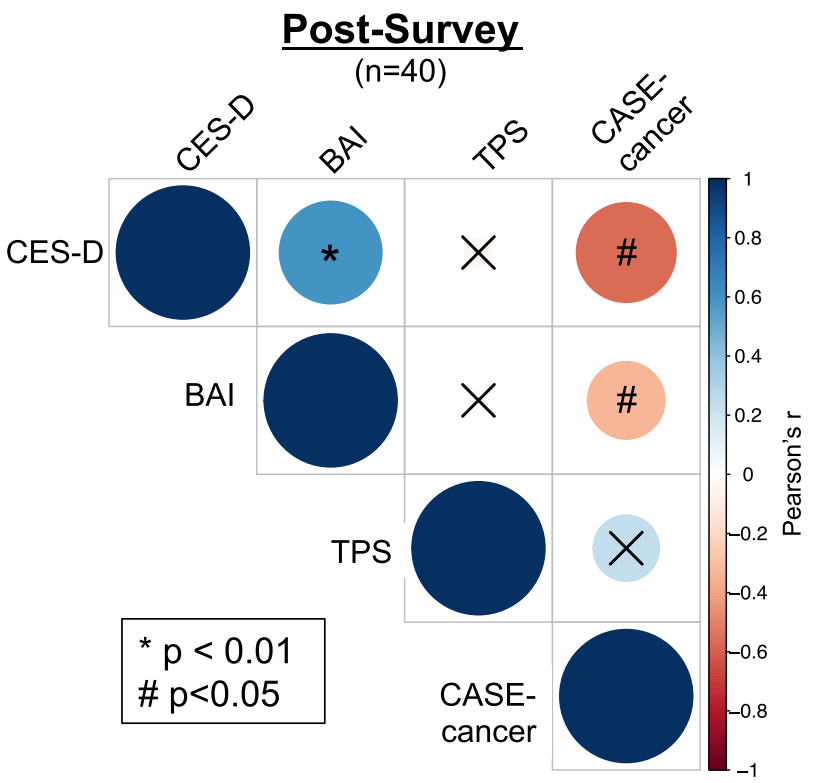

Fig. 2 Assessment and Change in Validated Psychological Metrics. Four validated psychosocial measures were assessed in patients at study entry: Center for Epidemiologic Studies Depression Scale (CES-D) [23], Beck Anxiety Inventory (BAI) [24], Trust in Physicians/Providers Scale (TPS) [25], and Communication and Attitudinal Self-Efficacy scale for cancer (CASE-cancer) [26]. a. Change in each measure was evaluated for those patients who completed both 'pre' and 'post' assessments ( $n=40$ patients). Direction of change in score is indicated in color as decrease (red), increase (green), and no change (blue). Association was assessed by Wilcoxon signed rank test. b/c. Correlation between patients' scores on each validated metric and all other assessed metrics was assessed at study entry (b; $n=58$ patients) and at end of study (c; $n=40$ patients). Direction correlation (Pearson's $r$ ) is indicated by the color of each dot (positive correlation in blue, negative correlation in red) and magnitude of correlation indicated by size of each dot (higher correlation is larger size). Associations that were not statistically significant are indicated with a black ' $x$ '

physician or other provider for information and $27.5 \%$ of patients used the internet, their physician, and/or other sources.

\section{Discussion}

As somatic genomic testing becomes increasingly adopted as part of standard care for $\mathrm{MBC}$, there is a critical need to assess its impact on patients outside of implications for clinical care. In this study, patients received identical genomic testing (Foundation Medicine) with results delivered immediately after progression, providing a consistent setting for evaluation of patient psychosocial factors, genetic understanding, and perception of genomic testing.

There is growing awareness of differences in genetic testing comprehension, as has been documented previously in 
Table 2 Subjective Knowledge about Genetics

1. You are confident in your ability to understand information about genetics.

\section{Post-test $N$ (\%)}

\begin{tabular}{|c|c|c|c|}
\hline $\begin{array}{l}\text { Pre-test } N \\
\text { (\%) }\end{array}$ & Disagree & Neutral & Agree \\
\hline Disagree & $4(10.3)$ & $3(7.7)$ & $0(0.0)$ \\
\hline Neutral & $0(0.0)$ & $\begin{array}{l}5 \\
(12.8)\end{array}$ & $4(10.3)$ \\
\hline Agree & $0(0.0)$ & $1(2.6)$ & $\begin{array}{l}22 \\
(56.4)\end{array}$ \\
\hline
\end{tabular}

2. It would be easy for you to get information about genetics if you wanted to.

\section{Post-test $N(\%)$}

Pre-test $N$

(\%)

Disagree

Neutral

Agree

Disagree Neutral Agree value*

0.80
McNemar's test $p$ - value*

0.19
Table 2 Subjective Knowledge about Genetics (Continued) 6. You would be able to explain to others how genes affect health.

\begin{tabular}{lllll} 
& \multicolumn{2}{c}{ Post-test $\boldsymbol{N}(\%)$} & $\begin{array}{l}\text { McNemar's test } \boldsymbol{p} \text { - } \\
\text { value* }\end{array}$ \\
$\begin{array}{l}\text { Pre-test } \boldsymbol{N} \\
\text { (\%) }\end{array}$ & Disagree & Neutral & Agree & $\mathbf{0 . 0 4}$ \\
Disagree & $5(12.8)$ & $\begin{array}{l}6 \\
(15.4)\end{array}$ & $1(2.6)$ \\
Neutral & $2(5.1)$ & $\begin{array}{l}6 \\
(15.4)\end{array}$ & $6(15.4)$ \\
& & & \\
Agree & $2(5.1)$ & $0(0.0)$ & 11 \\
& & & $(28.2)$
\end{tabular}

3. You would be able to understand information about how genes can affect your health.

\begin{tabular}{llll} 
& \multicolumn{2}{l}{ Post-test $\boldsymbol{N}(\%)$} & \\
$\begin{array}{l}\text { Pre-test } \boldsymbol{N} \\
\text { (\%) }\end{array}$ & Disagree & Neutral & Agree \\
Disagree & $1(2.6)$ & $1(2.6)$ & $0(0.0)$ \\
Neutral & $0(0.0)$ & 4 & $0(0.0)$ \\
& & $(10.3)$ & \\
Agree & $1(2.6)$ & 4 & 28 \\
& & $(10.3)$ & $(71.8)$
\end{tabular}

McNemar's test $p$ value*

0.11 disease generally.

\begin{tabular}{llll} 
& \multicolumn{2}{l}{ Post-test $\boldsymbol{N}(\%)$} & \\
$\begin{array}{l}\text { Pre-test } \boldsymbol{N} \\
\text { (\%) }\end{array}$ & Disagree & Neutral & Agree \\
Disagree & $3(9.1)$ & $0(0.0)$ & $1(3.0)$ \\
Neutral & $2(6.1)$ & 4 & $3(9.1)$ \\
& & $(12.1)$ & \\
Agree & $0(0.0)$ & $0(0.0)$ & 20 \\
& & & $(60.6)$
\end{tabular}

5. You have a good idea about how your own genetic makeup might affect your risk for disease.

\section{Post-test $N(\%)$}

Pre-test $N$

(\%)

$\begin{array}{llll}\text { Disagree } & 3(7.9) & 2(5.3) & 0(0.0) \\ \text { Neutral } & 1(2.6) & 5 & 5(13.2) \\ & & (13.2) & \\ \text { Agree } & 0(0.0) & 5 & 17 \\ & & (13.2) & (44.7)\end{array}$

\section{McNemar's test $p$ -} value*

0.11
Table 3 Objective Knowledge about Genetics

\begin{tabular}{|c|c|c|}
\hline \multicolumn{3}{|c|}{ 1. It is possible to see a gene with the naked eye. (False). } \\
\hline $\begin{array}{l}\text { Pre-test \% correct ( } N= \\
\text { 58) }\end{array}$ & $\begin{array}{l}\text { Post-test \% correct ( } N= \\
40 \text { ) }\end{array}$ & $\begin{array}{l}\text { McNemar's test } p^{-} \\
\text {value } \\
(N=40)\end{array}$ \\
\hline 77.2 & 87.2 & 0.04 \\
\hline \multicolumn{3}{|c|}{ 2. Healthy parents can have a child with a hereditary disease. (True) } \\
\hline $\begin{array}{l}\text { Pre-test \% correct ( } N= \\
\text { 58) }\end{array}$ & $\begin{array}{l}\text { Post-test \% correct ( } N= \\
40 \text { ) }\end{array}$ & $\begin{array}{l}\text { McNemar's test } p \text { - } \\
\text { value } \\
(N=40)\end{array}$ \\
\hline 92.7 & 92.3 & 0.32 \\
\hline
\end{tabular}

3. The carrier of a disease gene may be completely healthy. (True)

Pre-test $\%$ correct $(N=$ Post-test $\%$ correct $(N=$ McNemar's test $p$ 58) 40) value

$(N=40)$
91.2
87.2
$>0.99$

4. Genes are inside cells. (True)

Pre-test $\%$ correct $(N=$ Post-test $\%$ correct $(N=$ McNemar's test $p$ -

$\begin{array}{ll}\text { 58) } & \text { value } \\ & (N=40)\end{array}$
60.7
59.0
$>0.99$

5. A gene is a piece of DNA. (True)
Pre-test $\%$ correct $(N=$ Post-test $\%$ correct $(N=$ McNemar's test $p$ - 58)
40) value
$(N=40)$
70.2
61.5
0.48

6. All body parts have all of the same genes. (True)

Pre-test \% correct $(N=$ Post-test $\%$ correct $(N=$ McNemar's test $p$ 58)

$\begin{array}{ll}40) & \text { value } \\ & (N=40)\end{array}$
33.3
28.2
0.56

7. A person's race and ethnicity can affect how likely they are to get a disease. (True)

Pre-test $\%$ correct $(N=$ Post-test $\%$ correct $(N=$ McNemar's test $p$ 58)

40)

value

80.7

89.7

0.03 


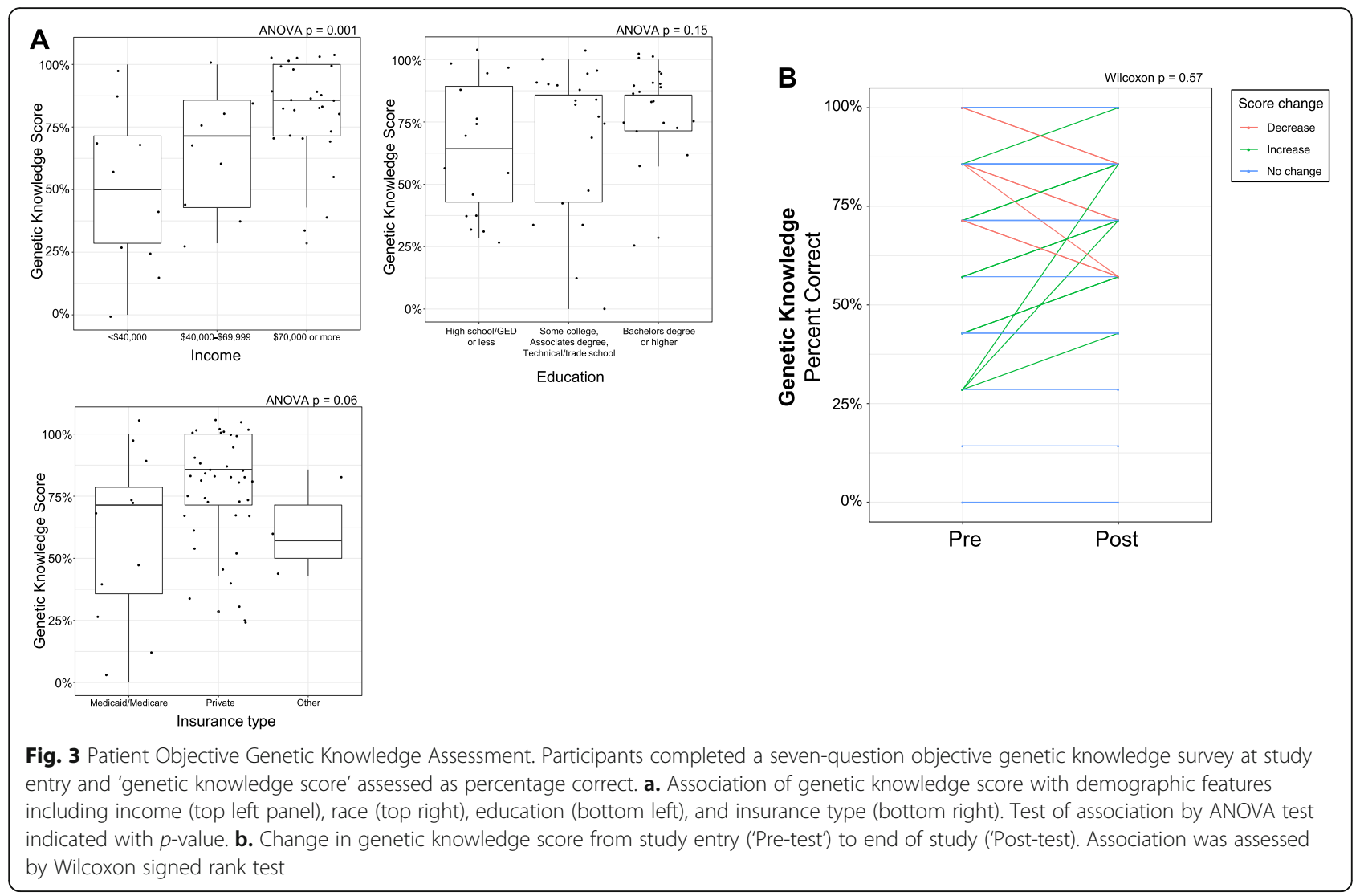

the scope of germline genomic testing and race [28], and in this study, patient understanding of somatic genomic testing showed substantial variability. We used a non-validated 7-question metric based on a previously published survey [27] to assess genetic knowledge and this demonstrated a wide range of baseline understanding of genetics, with a range 0 to $100 \%$ of questions answered correctly in both pre- and post-test. This suggests that providers cannot assume any baseline knowledge of genetics when offering somatic genomic tumor testing. In terms of sociodemographic patient factors, participants who identified as nonwhite and participants with lower annual incomes had significantly lower baseline genetic comprehension, which is consistent with previous findings $[28,29]$. The limited sample size prevented evaluation of possible confounders, such as literacy, numeracy, and other metrics of socioeconomic status. We also wish to note that performing this analysis as white vs. non-white due to statistical constraints introduced by our small sample size does limit the strength of our results. It is important to note that education was not associated with baseline genetic knowledge, possibly skewed by the fact that the observed cohort was predominantly well-educated, limiting our ability to detect an association.

We hypothesized that patients' genetic knowledge may improve over their time on a prospective clinical trial of somatic genomic testing. There was no formal educational intervention as part of this study but during the informed consent process, patients received information on the nature of genetics and genetic testing, the information this testing could yield, and how that information could impact care. However, our data do not demonstrate any significant change in genetic comprehension from pre- to post-study. While objective genetic comprehension remained stable, patients' self-assessed ability to explain the relationship between genetics and health to others increased. This suggests a potentially inflated self-appraised ability and understanding, an area explored in the perception of patients' health and in students' assessment of knowledge, but not before in the context of patient health education to our knowledge [30]. There is evidence that information seeking can falsely increase a patient's subjective knowledge within the domain of genetic testing and we did see that most patients (85\%) sought information after their genomic testing [31]. It is important for physicians to be aware of this phenomenon because it may influence patients concealing health illiteracy [32]. Collectively, these findings reinforce the need for interventions to educate patients about their care or research studies and to 
target health literacy, particularly in the informed consent process for genetic/genomic testing.

To further investigate patient understanding of genomic testing [14-17], we evaluated exploratory questions regarding patient motivations, expectations, perceived risks, and perceived benefits. Notably, the majority of patients mistakenly believed that the NGS test results would explain how they could change their own behavior to lower their risk of disease and that the results would help them to seek medical attention to reduce disease risk. This demonstration of advanced cancer patients' unrealistic expectations of somatic genomic testing corroborates and further informs recent study findings that indicate patients undergoing somatic tumor genomic testing may mistake its capabilities for germline genomic testing $[14,15,17,33]$.

Importantly, patients' misconceptions about tumor genomic testing has been linked to negative psychosocial effects, including feelings of anxiety, disappointment, guilt, and feelings of abandonement [14, 15, 17]. In addition, in there has been a growing focus on mental health within cancer care because of the preliminary documentation of associations between depression and worse survival outcomes in women with advanced breast cancer [34]. Although still disputed, it has been hypothesized that psychological distress, including anxiety and depression, may elicit disease progression by interfering with the hypothalamic pituitary adrenal axis [35]. While further study is warranted to explore the relationship between psychological health and clinical outcomes, we began to explore the impact of tumor genomic testing on emotional wellbeing. Our cohort of participants had a slightly higher average CES-D depression score (13.6) at baseline in comparison to another trial evaluating depressive-symptoms alone in MBC, which had an average CES-D score of 11.1 [34]. A recent meta-analysis assessed depression in patients with breast cancer worldwide and found that depression was prevalent in 32.2\% of patients, similar to our study population with a prevalence of $38 \%$ with a CES-D score reflective of depression. In terms of anxiety, $47 \%$ of women displayed anxiety in our cohort [36]. In comparison to other advanced cancer types, such as patients with late stage ovarian cancer with a mean BAI score of 16.88-19 at baseline, our cohort had less severe anxiety on average (mean score of 11.3). However, our study cohort had a slightly greater proportion of patients who experienced at least mild anxiety (47\%) in comparison to patients with late stage ovarian cancer (44\%) [37]. Interestingly, anxiety and depression positively correlated in our study, indicating that psychosocial interventions for MBC should be comprehensive and not only focus on depression or anxiety. Luckily, patients' mental health status remained rather stable over the duration of the study, indicating that tumor genomic testing does not significantly affect anxiety or depression.

This is the first study to our knowledge to evaluate MBC patients' trust in their doctor using the validated TPS metric. Patients' trust in their medical oncologist remained stable over the duration of the study with an average TPS score of 48.4-49.4 (pre- and post-survey, respectively). This is higher than other documented TPS study scores in rheumatic disease and primary care patients which ranged between 41.9 and 45.7, respectively $[38,39]$. The stability of the TPS score was interesting given our prior finding that patients lost confidence in their cancer treatment success after undergoing the genomic testing [19]. This previous finding was especially apparent in patients whose subsequent treatment was not supported by the FM test [19]. It is especially important to note and emphasize that a patient's subsequent cancer treatment was or was not supported directly by the FM test did not impact their depression, anxiety, trust in physician, or self-efficacy scores. In addition, a lack of correlation between the number of therapies recommended by the FM test and depression, anxiety, trust in physician, self-efficacy, and genetic knowledge scores further supports that the tumor genomic test does not harbor the possibility of harming patients more or less based on its clinically-oriented results.

Self-efficacy in cancer care, defined as patients' confidence in their ability to acquire information about their cancer care, understand their cancer care, and continue to have a positive psychological affect during their care, trended downward from before to after genomic testing. Within the context of CASE-cancer, this suggests that patients lose confidence in their perceived capacity to navigate their cancer care from before to after the NGS testing. One possible explanation of this finding is that patients required greater education from their physicians on tumor genomic testing in order to feel empowered to seek and understand information about the NGS. Because increased self-efficacy, amongst other patient psychological factors and communication styles with their physician, is correlated with better disease outcomes, future study of ways to preserve patient self-efficacy is needed [40]. One potential method to preserve patient self-efficacy is through increased patient education and empowering supportive interventions [40-42].

As genomic testing becomes increasingly integrated into clinical care, it is important to evaluate how the translation of this valuable information holistically affects patients. While other studies are beginning to evaluate patient comprehension and perceptions of genomic testing in other disease groups [43], this exploration in cancer care has primarily focused on germline genetic testing [44-46]. These studies still support the 
need for greater attention on the translation of genomic testing into clinical care, as similar concerns about patient misconceptions about results and what the tests offer are apparent [43].

Our study does have limitations. There was a relatively limited sample size, partly due to a lower response rate of 58\% (58/100). This limited sample size unfortunately curtailed our ability to assess the racial and ethnic background of our participants beyond that of "white" vs. "non-white" and the current analysis does not have significant power. In addition, we note that the grouping of multiple racial and ethnic groups collectively as "nonwhite" in "white" vs. "non-white" analyses is not ideal and limits the conclusions we can draw. A future analysis that is focused on the experiences of subjects of minority backgrounds is warranted. The secondary analysis still offers unique insight into critical areas regarding the translation of somatic genomic testing into clinical care because of its longitudinal design and utilization of multiple metrics. It is important to note that our use of nonvalidated metrics to evaluate patient comprehension of genetics, subjective perception of genetic knowledge, or patient perceived risks and benefits of genetic testing does limit our study. Further, the single-center design also supports the need for further exploration of these areas.

\section{Conclusions}

In conclusion, this is the first study to longitudinally evaluate the role of depression, anxiety, trust in physician, self-efficacy, objective genetic knowledge, subjective assessment of genetic knowledge, motivations and expectations in regards to somatic genomic testing in a single advanced cancer population. Further exploration of these areas in a larger study are warranted and will hopefully inform how to best support patients who are undergoing tumor genomic testing by better explaining the role of NGS in cancer care through the creation of patient-centered education initiatives.

\section{Supplementary information}

Supplementary information accompanies this paper at https://doi.org/10. 1186/s12885-020-06905-2.

Additional file 1: Table S1. Mixed Effects Model Table S2. Patient Motivations and Perceived Risks/Benefits Figure S1. Association of Validated Depression, Anxiety, Physician Trust, and Self-Efficacy Measures with Patient Demographic Features.

\footnotetext{
Abbreviations

BAl: Beck Anxiety Inventory; CASE-cancer: Communication and Attitudinal Self-Efficacy scale for cancer; CES-D: Center for Epidemiologic Studies Depression Scale; DNA: Deoxyribonucleic acid; ECOG: Eastern Cooperative Oncology Group; ER: Estrogen receptor; ESRI1: Estrogen Receptor 1; FM: Foundation Medicine; GED: General educational development; HER2: Human epidermal growth factor receptor 2; MBC: Metastatic breast cancer; NCl: National Cancer Institute; NGS: Next-generation sequencing;
}

PIK3CA: Phosphatidylinositol-4,5-Bisphosphate 3-Kinase Catalytic Subunit Alpha; PR: Progesterone receptor; TNBC: Triple negative breast cancer; TPS: Trust in Physicians/Providers Scale; TNBC: Triple negative breast cancer

\section{Acknowledgements}

The authors would like to acknowledge the clinical support of Catherine Carson, Celia Garr, Krysten Brown, and Katherine Tyson.

\section{Authors' contributions}

Study concept and design: RR, MAR, SG, JLC, CLS, ERM, RP, BR, MBL. Data collection: RR, SG, MAC, AMN, SS, JVD, RW, NW, CLS, ERM, BR, MBL. Data analysis and interpretation: EJA, SA, RR, KAC, MAR, RP, AET, LS, BR, CNL, MBL, DGS. Manuscript preparation: EJA, SA, KAC, MAR, MBL, DGS. All authors have read and approved the manuscript.

\section{Funding}

This work was supported through grants from Pelotonia for data anslyses and interpretation (D.G.S, M.B.L.), the Stephanie Spielman Fund for data analyses (D.G.S.) and Foundation Medicine, Inc. for genomic testing.

\section{Availability of data and materials}

All data generated or analyzed during this study are included in this published article.

\section{Ethics approval and consent to participate}

All procedures performed in studies involving human participants were in accordance with the ethical standards of the institutional and/or national research committee and with the 1964 Helsinki declaration and its later amendments or comparable ethical standards. All patients completed written informed consent, which included a description of the somatic genomic testing and discussion of study risks and benefits with their treating physician. This study was approved by the Ohio State University Institutional Review Board. Study reference numbers: OSU\#2012C0110 and NCT01987726.

\section{Consent for publication}

All authors agree with publication. All patient identifying information has been anonymised and there are no identifying images used within the manuscript.

\section{Competing interests}

The funding sources for the study, including Foundation Medicine, Inc., were not involved in the collection, analysis, or interpretation of the data. RW has received research support from Acerta and Astra Zeneca and served on advisory boards for PUMA and Pfizer. Drs. Stover, Lustberg, and Lee had full access to all the data in the study and had final responsibility for the decision to submit for publication.

\section{Author details}

${ }^{1}$ The Ohio State University Comprehensive Cancer Center, Arthur G. James Cancer Hospital and Richard J. Solove Research Institute, Columbus, OH, USA. ${ }^{2}$ Division of Medical Oncology, Ohio State University College of Medicine, Columbus, OH, USA. ${ }^{3}$ Division of Hospital Medicine, Ohio State University College of Medicine, Columbus, OH, USA. ${ }^{4}$ Center for Biostatistics, Department of Biomedical Informatics, The Ohio State University, Columbus, $\mathrm{OH}$, USA. ${ }^{5}$ Stefanie Spielman Comprehensive Breast Center, 1145 Olentangy River Rd, Columbus, OH, USA. ${ }^{6}$ Mount Sinai, New York, NY, USA. ${ }^{7}$ Columbus Oncology, Columbus, OH, USA. ${ }^{8}$ Department of Cancer Biology \& Genetics and Department of Internal Medicine, Division of Human Cancer Genetics, Ohio State University College of Medicine, Columbus, OH, USA. ${ }^{9}$ Department of Plastic and Reconstructive Surgery, College of Medicine, The Ohio State University, $\mathrm{OH}$, Columbus, USA. ${ }^{10}$ Division of Health Services Management and Policy, College of Public Health, The Ohio State University, Columbus, $\mathrm{OH}$, USA. ${ }^{11}$ Stefanie Spielman Comprehensive Breast Center, Ohio State University Comprehensive Cancer Center, Biomedical Research Tower, Room 512, Columbus, $\mathrm{OH}$ 43210, USA. 
Received: 6 December 2019 Accepted: 26 April 2020

Published online: 06 May 2020

\section{References}

1. Mariotto $A B$, Etzioni $R$, Hurlbert M, et al. Estimation of the number of women living with metastatic breast Cancer in the United States. Cancer Epidemiol Biomark Prev. 2017;26:809-15.

2. Chandarlapaty S, Chen D, He W, et al. Prevalence of ESR1 mutations in cellfree DNA and outcomes in metastatic breast Cancer: a secondary analysis of the BOLERO-2 clinical trial. JAMA Oncol. 2016;2:1310-5.

3. Fribbens $C$, O'Leary B, Kilburn $L$, et al. Plasma ESR1 mutations and the treatment of estrogen receptor-positive advanced breast Cancer. J Clin Oncol. 2016:34:2961-8

4. Razavi P, Chang MT, Xu G, et al. The Genomic Landscape of EndocrineResistant Advanced Breast Cancers. Cancer Cell. 2018;34:427-438 e6.

5. Condorelli R, Mosele F, Verret B, et al. Genomic alterations in breast cancer: level of evidence for actionability according to ESMO scale for clinical Actionability of molecular targets (ESCAT). Ann Oncol. 2019.

6. Andre F, Ciruelos E, Rubovszky G, et al. Alpelisib for PIK3CA-mutated, Hormone Receptor-Positive Advanced Breast Cancer. N Engl J Med. 2019; 380:1929-40.

7. Arnedos M, Vicier C, Loi S, et al. Precision medicine for metastatic breast cancer--limitations and solutions. Nat Rev Clin Oncol. 2015;12:693-704.

8. Dancey JE, Bedard PL, Onetto N, et al. The genetic basis for cancer treatment decisions. Cell. 2012;148:409-20.

9. Cheng DT, Mitchell TN, Zehir A, et al. Memorial Sloan Kettering-integrated mutation profiling of actionable Cancer targets (MSK-IMPACT): a hybridization capture-based next-generation sequencing clinical assay for solid tumor molecular oncology. J Mol Diagn. 2015;17:251-64.

10. Russnes HG, Navin N, Hicks J, et al. Insight into the heterogeneity of breast cancer through next-generation sequencing. J Clin Invest. 2011;121:3810-8.

11. Chin L, Andersen JN, Futreal PA. Cancer genomics: from discovery science to personalized medicine. Nat Med. 2011;17:297-303.

12. Berg JS, Khoury MJ, Evans JP. Deploying whole genome sequencing in clinical practice and public health: meeting the challenge one bin at a time. Genet Med. 2011;13:499-504

13. Gray SW, Park ER, Najita J, et al. Oncologists' and cancer patients' views on whole-exome sequencing and incidental findings: results from the CanSeq study. Genet Med. 2016:18:1011-9.

14. Miller FA, Hayeems RZ, Bytautas JP, et al. Testing personalized medicine: patient and physician expectations of next-generation genomic sequencing in late-stage cancer care. Eur J Hum Genet. 2014;22:391-5.

15. Gray SW, Hicks-Courant K, Lathan CS, et al. Attitudes of patients with cancer about personalized medicine and somatic genetic testing. J Oncol Pract. 2012:8:329-35, 2 p following 335.

16. Liang R, Meiser B, Smith S, Kasparian NA, Lewis CR, et al. Advanced cancer patients' attitudes towards, and experiences with, screening for somatic mutations in tumours: a qualitative study. Eur J Cancer Care. 2016:1-11. https://www.ncbi.nlm.nih.gov/pubmed/27730730.

17. Pellegrini I, Rapti M, Extra JM, et al. Tailored chemotherapy based on tumour gene expression analysis: breast cancer patients' misinterpretations and positive attitudes. Eur J Cancer Care (Engl). 2012;21:242-50.

18. Foundation Medicine Inc. FM. 2018. https://www.foundationmedicine.com/ genomic-testing/foundation-one-cdx. Accessed 28 Nov 2018.

19. Stover DG, Reinbolt RE, Adams EJ, Asad S, Tolliver K, Abdel-Rasoul M, Timmers CD, Gillespie S, Chen JL, Ali SM, Webster J, Collier KA, Cherian MA, Noonan AM, Sardesai S, VanDeusen J, Wesolowski R, Williams N, Lee CN, Shapiro CL, Macrae ER, Ramaswamy B, Lustberg MB. Prospective DecisionAnalysis Study of Clinical Genomic Testing in Metastatic Breast Cancer: Impact on Outcomes and Patient Perceptions. JCO Precision Oncol. 2019:111. https://ascopubs.org/doi/10.1200/PO.19.00090.

20. Arver B, Haegermark A, Platten U, et al. Evaluation of psychosocial effects of pre-symptomatic testing for breast/ovarian and colon cancer pre-disposing genes: a 12-month follow-up. Familial Cancer. 2004;3:109-16.

21. Collins VR, Meiser B, Ukoumunne $\mathrm{OC}$, et al. The impact of predictive genetic testing for hereditary nonpolyposis colorectal cancer: three years after testing. Genet Med. 2007;9:290-7.

22. Ertmanski S, Metcalfe K, Trempala J, et al. Identification of patients at high risk of psychological distress after BRCA1 genetic testing. Genet Test Mol Biomarkers. 2009;13:325-30.
23. Radloff LS. The CES-D scale: a self-report depression scale for research in the general population. Appl Psychol Measure. 1977;1:385-401.

24. Beck AT, Epstein N, Brown G, et al. An inventory for measuring clinical anxiety: psychometric properties. J Consult Clin Psychol. 1988;56:893-7.

25. Anderson LA, Dedrick RF. Development of the Trust in Physician scale: a measure to assess interpersonal trust in patient-physician relationships. Psychol Rep. 1990;67:1091-100.

26. Wolf MS, Chang, C.H., Davis, T., Makoul, G.: Development and validation of the communication and Attudinal self-efficacy scale for cancer (CASEcancer). Patient Educ Couns 57:333-341, 2005

27. Fitzgerald-Butt SM, Bodine A, Fry KM, et al. Measuring genetic knowledge: a brief survey instrument for adolescents and adults. Clin Genet. 2016:89:235-43.

28. Singer E, Antonucci T, Van Hoewyk J. Racial and ethnic variations in knowledge and attitudes about genetic testing. Genet Test. 2004;8:31-43.

29. Rini C, Henderson GE, Evans JP, et al. Genomic knowledge in the context of diagnostic exome sequencing: changes over time, persistent subgroup differences, and associations with psychological sequencing outcomes. Genet Med. 2020;22:60-8.

30. Dunning-Davies J. Finding evidence for black holes. Science. 2004;305:1238; author reply 1238.

31. DiMaso SN. Pre-appointment information seeking and scanning behaviors in individuals scheduled for cancer genetics consultations. (Electronic Thesis or Dissertation). Retrieved from https://etd.ohiolinkedu/.

32. Graham S, Brookey J. Do patients understand? Perm J. 2008;12:67-9.

33. Roberts JS, Gornick MC, Le LQ, et al. Next-generation sequencing in precision oncology: patient understanding and expectations. Cancer Med. 2019;8:227-37.

34. Giese-Davis J, Collie K, Rancourt KM, et al. Decrease in depression symptoms is associated with longer survival in patients with metastatic breast cancer: a secondary analysis. J Clin Oncol. 2011;29:413-20.

35. Spiegel D, Giese-Davis J. Depression and cancer: mechanisms and disease progression. Biol Psychiatry. 2003:54:269-82.

36. Pilevarzadeh M, Amirshahi M, Afsargharehbagh $\mathrm{R}$, et al. Global prevalence of depression among breast cancer patients: a systematic review and metaanalysis. Breast Cancer Res Treat. 2019;176:519-33.

37. Alexandre J, Brown C, Coeffic D, et al. CA-125 can be part of the tumour evaluation criteria in ovarian cancer trials: experience of the GCIG CALYPSO trial. Br J Cancer. 2012:106:633-7.

38. Freburger JK, Callahan LF, Currey SS, et al. Use of the Trust in Physician Scale in patients with rheumatic disease: psychometric properties and correlates of trust in the rheumatologist. Arthritis Rheum. 2003:49:51-8.

39. Mainous AG 3rd, Baker R, Love MM, et al. Continuity of care and trust in one's physician: evidence from primary care in the United States and the United Kingdom. Fam Med. 2001;33:22-7.

40. Street RL Jr, Makoul G, Arora NK, et al. How does communication heal? Pathways linking clinician-patient communication to health outcomes. Patient Educ Couns. 2009:74:295-301.

41. Pellino T, Tluczek A, Collins $M$, et al. Increasing self-efficacy through empowerment: preoperative education for orthopaedic patients. Orthop Nurs. 1998;17(48-51):54-9.

42. Anderson RM, Funnell MM, Butler PM, et al. Patient empowerment Results of a randomized controlled trial. Diabetes Care 1995:18:943-9.

43. Roberts JS, Robinson JO, Diamond PM, et al. Patient understanding of, satisfaction with, and perceived utility of whole-genome sequencing: findings from the MedSeq project. Genet Med. 2018;20:1069-76.

44. Heshka JT, Palleschi $\mathrm{C}$, Howley $\mathrm{H}$, et al. A systematic review of perceived risks, psychological and behavioral impacts of genetic testing. Genet Med. 2008;10:19-32.

45. Meiser B. Psychological impact of genetic testing for cancer susceptibility: an update of the literature. Psychooncology. 2005;14:1060-74.

46. Broadstock M, Michie S, Marteau T. Psychological consequences of predictive genetic testing: a systematic review. Eur J Hum Genet. 2000;8: $731-8$

\section{Publisher's Note}

Springer Nature remains neutral with regard to jurisdictional claims in published maps and institutional affiliations. 